of the six patients who survived hospitalization. All casepatients, but no non-case-patients, were exposed to 11.5year-old cellulose acetate dialyzers (all of these dialyzers were discarded by the hospital before the investigation). Laboratory investigation of field-retrieved 0 - to 13.6-yearold dialyzers of similar type indicated significant chemical degradation in the older membranes. In vivo injection of extracts of membrane degradation products produced iritis and hemorrhages in rabbits' eyes.

It was concluded that severe patient injury was associated with exposure to aged cellulose acetate membranes of dialyzers, allowing cellulose acetate degradation products to enter the blood. Clinicians should be aware that aged cellulose acetate membranes might cause severe adverse reactions.

FROM: Hutter JC, Kuehnert MJ, Wallis RR, Lucas $A D$, Sen $S$, Jarvis WR. Acute onset of decreased vision and hearing traced to hemodialysis treatment with aged dialyzers. JAMA 2000;283:2128-2134.

\section{Molecular Epidemiology of $S$ epidermidis in an NICU}

Coagulase-negative staphylococci, especially Staphylococcus epidermidis, are increasingly important nosocomial pathogens, particularly in critically ill neonates. Villari and coinvestigators, from the Department of Health and Preventive Sciences, University Federico, Naples, Italy, conducted a 3-year prospective surveillance of nosocomial infections in a neonatal intensive care unit (NICU) using traditional epidemiological methods, as well as molecular typing of microorganisms. The objectives of the study were (1) to quantify the impact of $S$ epidermidis on NICU-acquired infections; (2) to establish if these infections are caused by endemic clones or by incidentally occurring bacterial strains of this ubiquitous species; (3) to evaluate the use of different methods for the epidemiological typing of the isolates; and (4) to characterize the occurrence and the spread of staphylococci with decreased glycopeptide susceptibility.

The results showed that $S$ epidermidis is one of the leading causes of NICU-acquired infections and that the reduced glycopeptide susceptibility, if investigated by appropriate detection methods such as population analysis, is more common than is currently realized. Typing of isolates, which can be performed effectively through molecular techniques such as pulsed-field gel electrophoresis but not through antibiograms, showed that many of these infections are due to clonal dissemination and thus are potentially preventable by strict adherence to recommended infection control practices and the implementation of programs aimed toward the reduction of the unnecessary use of antibiotics. These strategies are also likely to have a significant impact on the frequency of the reduced susceptibility of staphylococci to glycopeptides, since this phenomenon appears to be determined either by more resistant clones transmitted from patient to patient or, to a lesser extent, by strains that become more resistant as a result of antibiotic pressure.

FROM: Villari P, Sarnataro C, Iacuzio L. Molecular epidemiology of Staphylococcus epidermidis in a neonatal intensive care unit over a three-year period. $J$ Clin Microbiol 2000;38:1740-1746.

\section{Growth of $S$ epidermidis and $P$ aeruginosa on Biomedical Polymers}

The infection risk of biomaterials implants varies between different materials and is determined by an interplay of adhesion and surface growth of the infecting organisms. Gottenbos and colleagues from the University of Groningen, The Netherlands, conducted a study that compared initial adhesion and surface growth of Staphylococcus epidermidis HBH(2) 102 and Pseudomonas aeruginosa $\mathrm{AK} 1$ on poly (dimethylsiloxane), Teflon, polyethylene, poly-propylene, polyurethane, poly(ethylene terephthalate), poly(methyl methacrylate), and glass. Initial adhesion was measured in situ in a parallel plate flow chamber with microorganisms suspended in phosphate-buffered saline, while subsequent surface growth was followed in full and in 20 times diluted growth medium. Initial adhesion of both bacterial strains was similar to all biomaterials. In full growth medium, generation times of surface growing $S$ epidermidis ranged from 17 to 38 minutes with no relation to wettability, whereas in diluted growth medium, generation times increased from 44 to 98 minutes with increasing surface wettability. For $P$ aeruginosa, no influence of surface wettability on generation times was observed, but generation times increased with decreasing desorption rates, maximal generation times being 47 minutes and minimal values down to 30 minutes. Generally, generation times of adhering bacteria were shorter than of planktonic bacteria.

The authors concluded that surface growth of initially adhering bacteria is influenced by biomaterials surface properties to a greater extent than initial adhesion.

FROM: Gottenbos B, van der Mei HC, Busscher HJ. Initial adhesion and surface growth of Staphylococcus epidermidis and Pseudomonas aeruginosa on biomedical polymers. J Biomed Mater Res 2000;50:208-214.

\section{Catheter-Associated UTIs Are Rarely Symptomatic}

Catheter-associated urinary tract infection (CA UTI) is the most common nosocomial infection, accounting for more than 1 million cases each year in US hospitals and nursing homes. Up to one half of the patients requiring an indwelling urethral catheter for 5 days or longer will develop bacteriuria or candiduria. Silent catheter-associated bacteriuria comprises a huge reservoir of antibioticresistant organisms in the hospital, particularly on the critical-care unit. Although there have been recommen- 
dations to treat CA UTIs only when they are symptomatic, the symptoms associated with CA UTI have not been clearly defined. Researchers at the University of Wisconsin Hospitals, Madison, reported on the findings of a study to determine the prevalence of signs and symptoms attributable to CA UTI and the relative contribution of CA UTI to nosocomial bloodstream infection (BSI).

In a prospective study, 1,497 newly catheterized patients were followed, and daily quantitative urine cultures and urine leukocyte counts were obtained. A detailed analysis was done of a subset of 1,024 patients, 89 of whom developed CA UTI with more than 103 colony-forming units per $\mathrm{mL}$, who did not have another potentially confounding site of infection besides the urinary tract.

There were 235 new cases of nosocomial CA UTI during the study period. More than $90 \%$ of the infected patients were asymptomatic; only 123 infections (52\%) were detected by patients' physicians using the hospital laboratory. In the subset analysis, there were no significant differences between patients with and without $\mathrm{CA}$ UTI in signs or symptoms commonly associated with UTI-fever, dysuria, urgency, or flank pain —or leukocytosis. Only 1 of the 235 episodes of CA UTI that were prospectively studied was unequivocally associated with secondary BSI.

CA UTIs are a major reservoir of antibiotic-resistant organisms in the hospital, but they are rarely symptomatic and infrequently cause BSI. Symptoms referable to the urinary tract, fever, or peripheral leukocytosis have little predictive value for the diagnosis of CA UTI.

FROM: Tambyah PA, Maki DG. Catheter-associated urinary tract infection is rarely symptomatic: a prospective study of 1,497 catheterized patients. Arch Intern Med 2000;160:678-682.

\section{Plague as Biological Weapon}

The Working Group on Civilian Biodefense has developed consensus-based recommendations for measures to be taken by medical and public health professionals following the use of plague as a biological weapon against a civilian population. The working group included 25 representatives from major academic medical centers and research, government, military, public health, and emergency-management institutions and agencies. The group developed a consensus statement supported by all working group members.

The statement concludes that an aerosolized plague weapon could cause fever, cough, chest pain, and hemoptysis, with signs consistent with severe pneumonia 1 to 6 days after exposure. Rapid evolution of disease would occur in the 2 to 4 days after symptom onset and would lead to septic shock, with high mortality without early treatment. Early treatment and prophylaxis with streptomycin or gentamicin or the tetracycline or fluoroquinolone classes of antimicrobials would be advised.

FROM: Inglesby TV, Dennis DT, Henderson DA, Bartlett JG, Ascher MS, Eitzen E, et al. Plague as a biologic weapon: Medical and public health management. Recommendations for Plague. JAMA 2000;283:2281-2290.

\section{Antimicrobial Susceptibility Testing of $P$ aeruginosa Isolates From CF Patients}

Pseudomonas aeruginosa is the most common pathogen infecting the lungs of patients with cystic fibrosis (CF). Improved antimicrobial chemotherapy has significantly increased the life expectancy of these patients. However, accurate susceptibility testing of $P$ aeruginosa isolates from CF sputum may be difficult, because the organisms are often mucoid and slow growing. Burns and coinvestigators, from the University of Washington, Seattle, reported on a study of $597 \mathrm{CF}$ isolates of $P$ aeruginos $a$ and examined the correlation of disk-diffusion and E-test (AB BIODISK, Solna, Sweden) results with a reference broth microdilution method. The rates of interpretive errors for 12 commonly used antipseudomonal antimicrobials were determined. The disk-diffusion method correlated well (zone diameter vs minimum inhibitory concentration [MIC]) for all of the agents tested. However, for mucoid isolates, correlation coefficients ( $\mathrm{r}$ values) for piperacillin, piperacillin-tazobactam, and meropenem were $<0.80$. The E-test correlation with reference broth microdilution results (MIC vs MIC) was acceptable for all of the agents tested, for both mucoid and nonmucoid isolates. Category interpretation errors were similar for the disk-diffusion and E-test methods, with 0.4 and $0.1 \%$, respectively, very major errors (false susceptibility) and 1.1 and $2.2 \%$ major errors (false resistance). Overall, both agar diffusion methods appear to be broadly acceptable for routine clinical use in susceptibility testing of $\mathrm{CF}$ isolates of $P$ aeruginosa.

FROM: Burns JL, Saiman L, Whittier S, Larone D, Krzewinski J, Liu $Z$, et al. Comparison of agar diffusion methodologies for antimicrobial susceptibility testing of Pseudomonas aeruginosa isolates from cystic fibrosis patients. J Clin Microbiol 2000;38:1818-1822. 